\title{
Werkzeuge und Instrumente in Kunstgeschichte und Technikanthropologie
}

Als das Wort »Werkzeug« im 12. Jahrhundert in der mittelhochdeutschen Volkssprache aufkam, hatte es einen ähnlichen Geltungsbereich wie das lateinische instrumentum, aus dem später »Instrument« hervorging: Es bezeichnete alles, was dem Mensch nützlich ist und von ihm für eine bestimmte Funktion hergestellt wurde. ${ }^{1}$ Die einst nahezu deckungsgleichen Begriffe haben sich jedoch auseinander entwickelt, so dass sie heute auf zwei verschiedene Gegenstandsgattungen bzw. Aktivitätsbereiche hinweisen. Im heutigen Sprachgebrauch sind Werkzeuge Dinge, die in der Hand gehalten werden, um auf ein formbares Material wie Holz, Stein oder Metall direkt einzuwirken oder um an etwas bereits Konstruiertem, zum Beispiel einem Motorrad, zu arbeiten. Instrumente dagegen sind zwar auch Dinge, mit denen man etwas tut, bevorzugt aber solche, die feiner oder komplizierter gebaut sind und in besonders gewürdigten Bereichen benutzt werden, wie Musik- und Wissenschaftsinstrumente. Nach dieser Unterscheidung, die sich besonders während des 19. Jahrhunderts durchsetzte, arbeiten auch Künstler eher mit Instrumenten als mit Werkzeugen (bzw. tools, outils, attrezzi), die gemeinhin Handwerkern zugeschrieben werden. ${ }^{2}$ Hierzu kommen noch die Begriffe "Maschine«, "Apparat« oder »technisches Gerät«, die ebenfalls auf eine lange Geschichte zurückblicken und auf andere, von der Mechanik und der Elektronik geprägte Dinge und Tätigkeitsfelder verweisen.

Aus diesen Beobachtungen ergeben sich vielfältige Aufgaben für die Kunstgeschichte als akademische Disziplin, die bei ihrer Konstituierung selbst von der Unterscheidung zwischen Werkzeug und Instrument geprägt wurde. Es sollte möglich sein, ausgehend von diesem Begriffspaar die abendländische Geschichte der Herstellung und Handhabung von Dingen zu erhellen, und dabei die Spezifizität von `Kunst in der Gesellschaft einzuschätzen. Dieses Vorhaben schließt die Erfassung eines weiten Spektrums historischer instrumenta mit ein, die zwar nicht Kunstwerke im tradierten Sinne sind, aber doch aufwendige Produkte des Könnens. Darüber hinaus stellt sich die Frage, was die Geschichte der Kunst zum breiten Feld der Forschungen über Technik beitragen kann. Dieser Ansatz wurde in jüngerer Zeit durch Untersuchungen 
zur technischen Funktion von Bildern eingeführt. ${ }^{3}$ Er soll hier vertieft werden, indem die Aufmerksamkeit auf Werkzeuge und Instrumente als Objekte, Prozesse und Repräsentationen von Kunst und Technik in historischer und interdisziplinärer Perspektive gerichtet wird.

\section{Objekte}

Werkzeuge und Instrumente sind strenggenommen keine Objekte. Wie es die Etymologie von objectum mit objicere (vor-werfen) nahelegt, stehen Objekte einem Subjekt gegenüber, sind getrennt von ihm und inaktiv. Werkzeuge und Instrumente dagegen stellt man sich primär in einer bestimmten Funktion vor, mit dem Subjekt verbunden. Dies wird evident, wenn man zum Beispiel sein mentales Bild eines Lassos überprüft: Dominierend ist nicht das gerollte Seil am Nagel an der Wand, sondern die animierte Form in der Luft. Erst durch Klassifizierung und Ausstellung werden Werkzeuge und Instrumente stillgestellt, ihrer primären Funktionen beraubt und letztendlich als Objekte im eigentlichen Sinne konstituiert. So werden sie als materielle Gebilde der Neugierde oder der Wissenschaft ausgeliefert. Diese Beobachtung lädt zu einem Streifzug durch die Geschichte der systematischen Sammlung von Werkzeugen und Instrumenten ein, wobei nach ihrem jeweiligen Status gefragt werden soll.

Einen ersten Kontext bilden dafür die fürstlichen Kabinette der Frühen Neuzeit. Dort sollten unter anderem, wie Samuel von Quiccheberg es in einem 1565 publizierten Traktat vorschlug, Instrumente (instrumenta) der Musik, der Mathematik, des Schreibens und Malens, der diversen künstlerischeren oder geläufigeren Künste ( $a$ b artificiosoribus artificibus usurpantur bzw. omnium artificum), der Chirurgie, der Jagd oder auch des Spielens versammelt werden. ${ }^{4}$ Die Zusammenkunft solcher Dinge an einem einzigen, autonomen Ort modifizierte den Umgang mit und das Verständnis von ihnen. So hat die mit innovativer Zahnstange und Kurbelgehäuse versehene sowie mit Intarsien und Ätzmalereien verzierte Drahtziehbank (Abb. 1), die 1565 für den persönlichen Gebrauch des Kurfürsten August von Sachsen (1553-1586) gefertigt wurde, sowohl in der ihre Stärke beweisenden Mechanik als auch in ihrer Kostbarkeit nur noch wenig mit dem instrumentum eines gewöhnlicheren Handwerkers zu tun. ${ }^{5}$ Ebenfalls geht das Sammeln von Musikinstrumenten mit der Entwicklung einer neuen Instrumentalmusik einher, welche sich in der zweiten Hälfte des 16. Jahrhunderts als eine eigene Gattung neben der Vokalmusik etablierte. Darüber hinaus ermöglichten die Dresdner Bestände Michael Praetorius, der unter anderem Kapellmeister des sächsischen Kurfürsten war, um 1620 die erste systematische Beschreibung und Analyse von Musikinstrumenten vorzulegen (Abb. 2). ${ }^{6}$ Außerdem entstanden vornehmlich an den Höfen des 17. Jahrhunderts, zusätzlich zu den tradierten Wissenschaftsinstrumenten wie etwa Zirkel, Lineal, Waage, Astrolab oder Uhr, neue Gerätschaften wie Fernrohr und Mikroskop, Thermometer und Barometer: Sie waren Teil von Expe- 


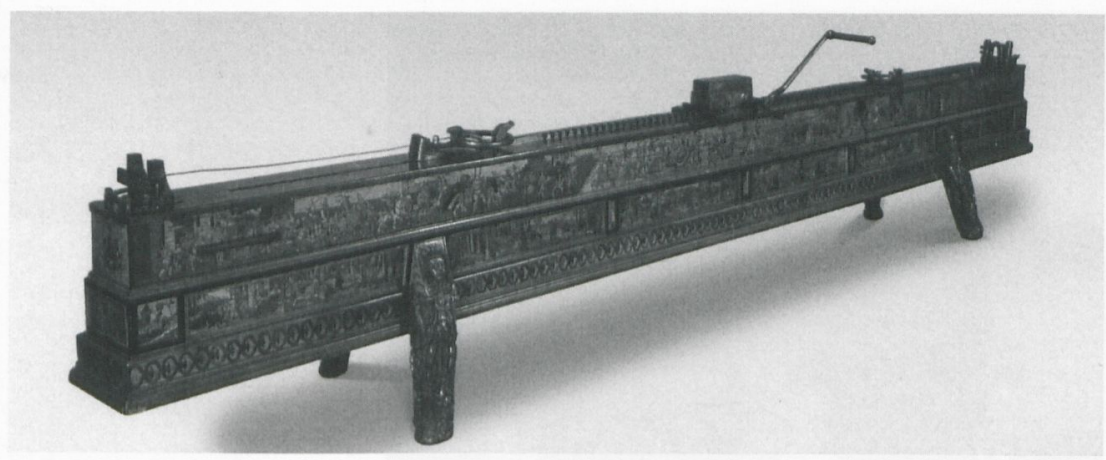

1. Drahtziehbank für den Kurfürsten August von Sachsen, Leonhard Danner, 1565.

Écouen, Musée national de la Renaissance

2. Tafel aus Michael Praetorius: Syntagma Musicum. De Organographia, Wolfenbüttel 1619-1620

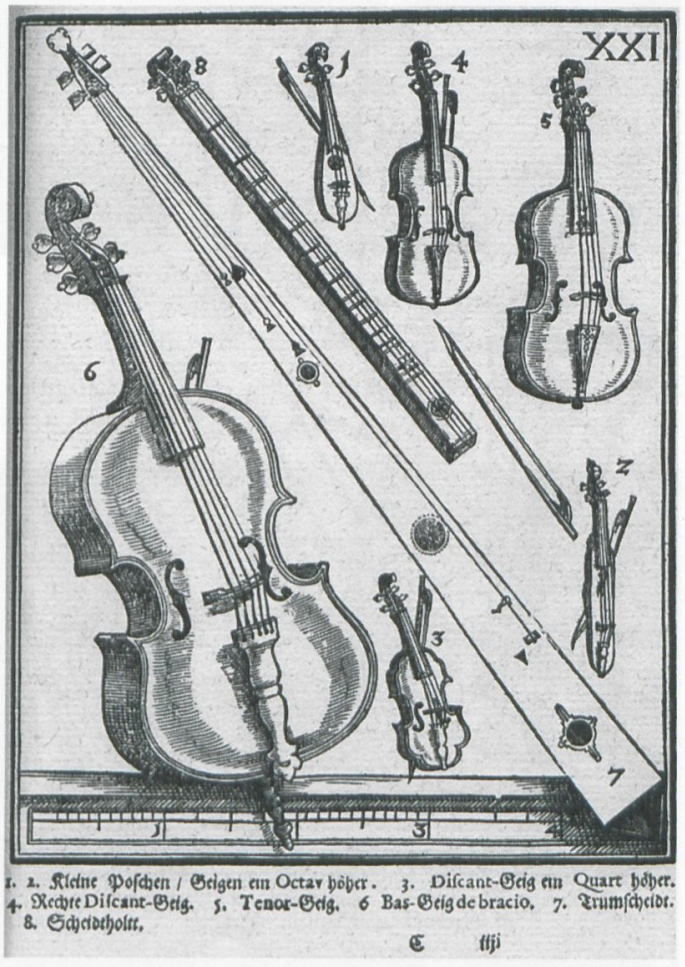

rimenten, mit denen neuartige Erkenntnisse über die Umwelt erzielt werden sollten. ${ }^{7}$ In der Folge der frühneuzeitlichen Faszination für operative Dinge ${ }^{8}$ treten zugleich Erzeugnisse auf, die gezielt den Instrumentenbegriff bis zur Funktionslosigkeit führen bzw. mit dessen Grenzen spielen: so zum Beispiel eine Kugel aus toskanischer pietra paesina, die der dänische Wissenschaftler 
PHILIPPE CORDEZ

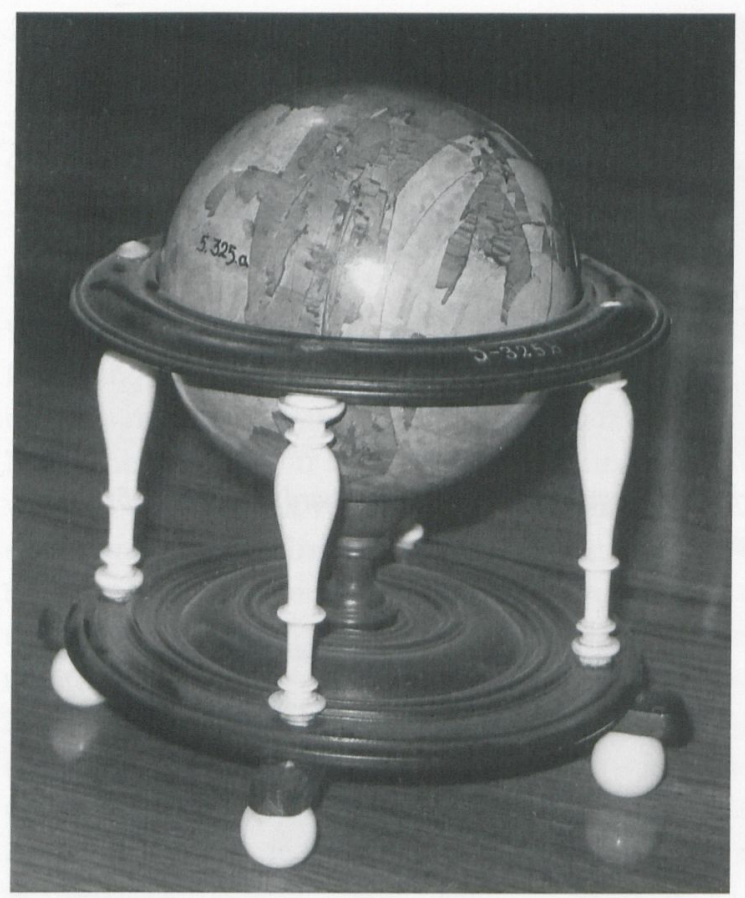

3. Kugel aus pietra paesina, montiert als Erdglobus für Olaus Wormius, um 1650. København, Kongens Kunstkammer

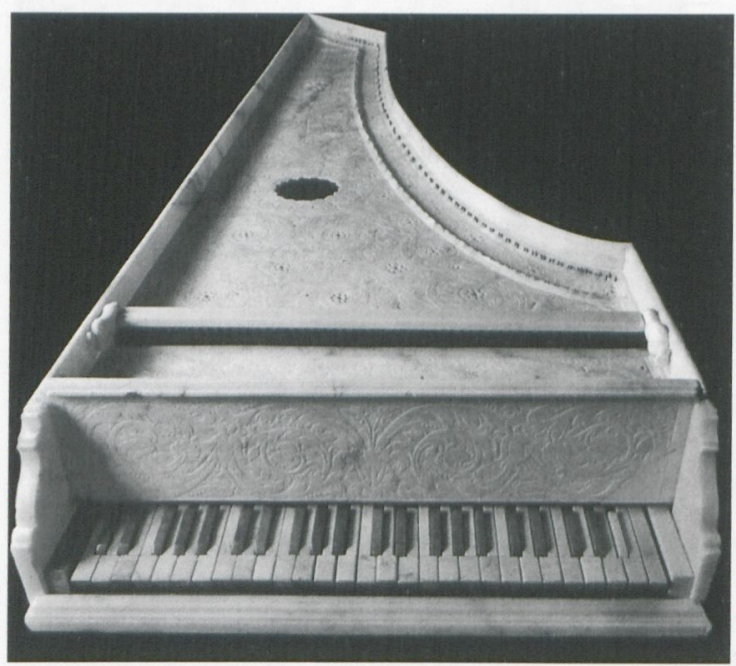

4. Cembalo aus Carrara-Marmor für den Herzog Francesco II. d'Este, Michele Antonio Grandi, 1687. Modena, Galleria Estense 
5. Maréchal Ferrantet Opérant, Outils, Tafel aus Denis Diderot $u$. Jean le Rond D'Alembert ( $\mathrm{Hg}$.): Encyclopédie ou Dictionnaire raisonné des sciences, des arts et des métiers, 28 Bde., Paris 1751-1772, Planches, Bd. 7

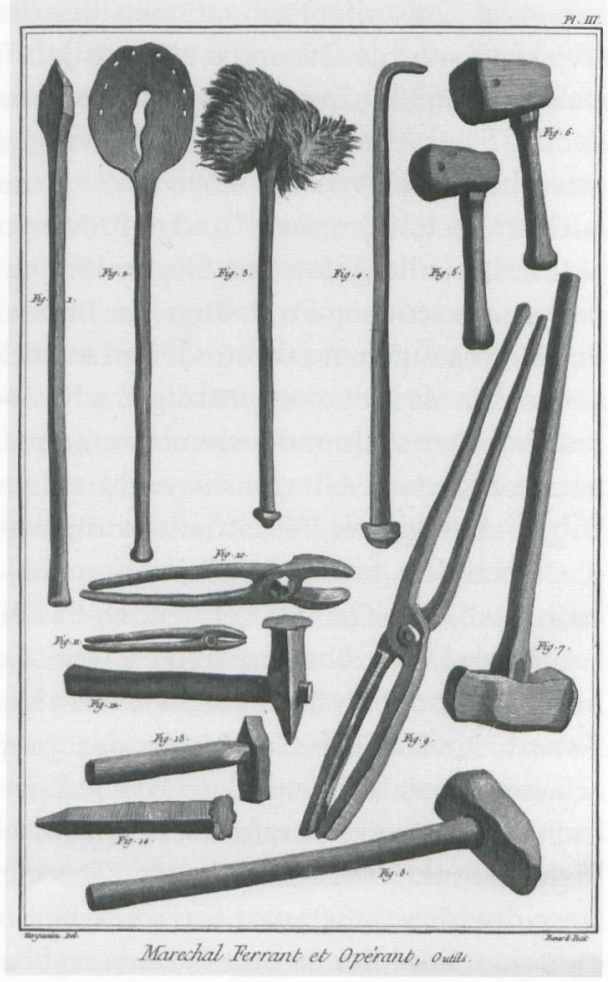

Olaus Wormius gegen 1650 wie einen Erdglobus montieren ließ (Abb. 3), wobei er sich zu Nutze machte, dass die natürliche Struktur dieses grünlichen und bräunlichen Gesteins das Liniengewirr kartographischer Konventionen evoziert, ${ }^{9}$ oder ein Cembalo, das 1687 für den Herzog Francesco II. d'Este in Modena aus Carrara-Marmor gemeißelt wurde (Abb. 4) und auf dem doch gespielt werden kann. ${ }^{10}$

Eine nächste wesentliche Etappe innerhalb der Sammlungsgeschichte von Werkzeugen und Instrumenten markieren ab der zweiten Hälfte des 19. Jahrhunderts die Kunstgewerbe- und Volkskundemuseen. Das technische Interesse der Frühen Neuzeit hatte sich mit großer Eigendynamik in der Industriellen Revolution weiter entfaltet. Bereits die zwischen 1751 und 1772 herausgegebene Encyclopédie ou Dictionnaire raisonné des sciences, des arts et des métiers hatte den Anspruch verfolgt, möglichst alle »Wissenschaften, Künste und Berufe « zu beschreiben, und aus dieser Motivation heraus Hunderte von Werkzeugen, Instrumenten und Maschinen in ihrem Funktionszusammenhang erläutert und anhand detaillierter Radierungen abgebildet (Abb. 5). ${ }^{11}$ Diese Demonstration des menschlichen Könnens wirkte als Innovationskatalysator. Sie fand eine Fortsetzung in den Lehrsammlungen des 1794 in Paris gegründeten Conservatoire des arts et métiers und später in zahlreichen Indus- 
trie- und Gewerbepräsentationen bei den Weltausstellungen, den großen Objektschauen des 19. und frühen 20. Jahrhunderts. Bereits die erste Weltausstellung, die 1851 in London stattfand, wies eine historische Sektion auf, aus der das South Kensington Museum hervorging, das zum Vorbild weiterer Kunstgewerbemuseen werden sollte. Diese Kunstgewerbemuseen verstanden sich auch als Technikmuseen ${ }^{12}$ und definierten sich zudem als zukunftsorientiert: Sie wollten alle Arten von Gegenständen zeigen, die im Gegensatz zu den neuen industriellen Produkten jene hohen Kunstfertigkeiten demonstrierten, die in vergangenen Jahrhunderten erreicht worden waren und noch in der neuen Ära als imitationswürdig erschienen. Volks- und Völkerkundemuseen präsentierten währenddessen bevorzugt die Erzeugnisse des ländlichen und handwerklichen Alltags, die nicht zuletzt angesichts der fortschreitenden Urbanisierung und Industrialisierung verstärkt auf Interesse stießen.

Neben dem neuen Terminus Kunstobjekt (bzw. objet d'art ${ }^{13}$ ) wurde dabei auch das Wort »Gerät « ${ }^{14}$ verwendet, das die von Menschen hergestellten und benutzten Dinge übergreifend erfasste. Gerät wies weniger auf die Ästhetik hin, als auf die zivilisatorische Relevanz. In diesem Sinne versuchten auch Kunsthistoriker die Geschichte der gesamten menschlichen Artefakte zu erfassen. So entwickelte Gottfried Semper (1803-1879), der selber an der englischen Kunstgewerbereform und an Museumsfragen beteiligt war, unter dem Begriff »Praktische Ästhetik« eine Theorie des Stils, die von einer Klassifizierung der Materialien und ihrer Bearbeitung ausging. ${ }^{15}$ An diesem Scharnier zwischen Funktionen und Formen etablierte sich auch der Ausdruck Volkskunst: Alois Riegl (1858-1905), langjähriger Kurator am Österreichischen Museum für Kunst und Industrie, betrachtete die entsprechenden Objekte in ihren sozialen Produktionsbedingungen, bevor er wenig später seine Gedanken zum »Kunstwollen« in Bezug auf die Formen aller Gegenstandsarten einer Epoche formulierte. ${ }^{16}$ Aby Warburg (1866-1929) entwickelte seinerseits eine Auffassung von Gerät, mit der er die materiellen Produktionen des Menschen als Befriedigung funktionaler wie auch ästhetischer Bedürfnisse gleichermaßen erfassen konnte. ${ }^{17}$ Henri Focillon (1881-1943) engagierte sich für ein umfassendes Studium des Menschen anhand aller seiner Objekte, ${ }^{18}$ und George Kubler (1912-1996) bemühte sich in dessen Nachfolge um eine Beschreibung des morphologischen Zusammenhanges aller möglichen Artefakte. ${ }^{19}$

\section{Prozesse}

Parallel zur Sammlungs- und Ausstellungspraxis und zum formorientierten Interesse hatte sich auch ein wissenschaftlicher Strang entwickelt, der die Werkzeuge und Instrumente weniger morphologisch als technologisch, also im Akt ihrer Verwendung, historisch erfassen und systematisch analysieren wollte. Die Entdeckung svorsintflutlicher Werkzeuge aus Feuerstein durch den Franzosen Jacques Boucher de Perthes (1788-1868) um die Mitte des 
19. Jahrhunderts ${ }^{20}$ sollte nicht nur das Wissen um die frühe Geschichte des Menschen revolutionieren, sondern zugleich das wissenschaftliche Verständnis von Werkzeugen und Instrumenten grundsätzlich bestimmen, zusammen mit ethnographischen, später auch primatologischen Beobachtungen. So ist der englische Generalleutnant Augustus Pitt Rivers (1827-1900) der erste, der in Anlehnung an die Evolutionstheorie Charles Darwins (1809-1882) seine umfangreiche Sammlung ethnographischer und archäologischer Artefakte funktionstypologisch und chronologisch ordnete, bevor er sie 1884 in ein nach ihm benanntes Museum in Oxford übertrug. ${ }^{21}$ Der deutsche Philosoph Ernst Kapp (1808-1896) veröffentlichte 1877 eine Entstehungsgeschichte der Cultur aus neuen Geschichtspunkten, in der er die philosophische Theorie der »Organprojektion « formulierte. ${ }^{22}$ Für die Entwicklung empirischer Forschungen wurde ein 1935 erschienener Aufsatz des französischen Soziologen und Ethnologen Marcel Mauss (1872-1950) zu den »Körpertechniken « entscheidend. ${ }^{23}$ Nach dem zweiten Weltkrieg legte der Ethnologe und Prähistoriker André LeroiGourhan (1911-1986) mehrere wegweisende Studien zur Anthropologie der Techniken vor ${ }^{24}$ und prägte zugleich einen Forschungszweig, der sich bis heute unter dem Namen technologie culturelle (»kulturelle Technikwissenschaft«) fortbildet. ${ }^{25}$ Solche Überlegungen wurden in dem jungen Feld der Soziologie der Wissenschaft und der Technik weiterentwickelt, ${ }^{26}$ in empirischen und theoretischen Arbeiten zur Sachkultur, ${ }^{27}$ oder auch in Studien der pragmatischen Philosophie ${ }^{28}$ und auf dem Gebiet der Politikwissenschaft. ${ }^{29}$ Die Technikphilosophie ${ }^{30}$ gewinnt mit der Technikfolgenabschätzung und -bewertung eine angewandte Dimension, die besonders für die Ausbildung an technischen Universitäten entscheidend ist. ${ }^{31}$ Schließlich bemühen sich auch ökologische Aktivisten darum, die Fragen der Technik ins Zentrum des öffentlichen Gesellschaftsinteresses zu rücken. ${ }^{32}$

Es fällt bereits in diesem kurzen Überblick auf, wie sehr die sozialwissenschaftlichen Forschungen zur Technik und damit auch die öffentliche Technikdebatte sich unabhängig von der Kunstgeschichte entwickelt haben. Sowohl die Kunstgeschichte, die sich zunächst vorwiegend der abendländischen Tradition gewidmet hat, als auch die Anthropologie der Kunst, ${ }^{33}$ die später aus außereuropäischen Forschungsfeldern hervorging, haben sich gemäß der seit dem 18. und 19. Jahrhundert in Europa dominierenden Auffassung von Kunst primär mit ästhetischen Phänomenen beschäftigt und dabei den Bereich der Technik vernachlässigt - obwohl das lateinische Wort ars den griechischen Terminus techne lediglich übersetzt und die Etymologie von "Kunst" als Ausdruck des Könnens ebenfalls der Technik naheliegt. Erst um die Mitte des 18. Jahrhunderts wurde in ars bzw. Kunst zwischen Technik und Ästhetik getrennt $^{34}$. Eine solche Situation führt heute zu der Herausforderung, die Kategorien von Kunst und Technik, wie sie seit der Gründung der akademischen Disziplinen tradiert worden sind, nun aus historischer Distanz zu betrachten, um jene Fachdivergenzen zu überwinden, die vielmehr dem idealistischen und kolonialen Weltbild des 19. Jahrhunderts entsprachen, als sie 
heute die aktualisierten Aufgaben der Sozialwissenschaften bedienen können. Über die sInterdisziplinarität ‘ hinaus, die notwendigerweise von älteren, trennenden Auffassungen bedingt ist, sollten für weitere Diskussionen neue und freiere, die einzelnen Fachgeschichten reflektierende smetadisziplinarische`Arbeitsfelder eröffnet werden. Technische Universitäten als konzeptionelle Orte künftiger Technikgestaltung und zugleich als Standorte sozialwissenschaftlicher Forschung und Lehre dürften hierfür eine entscheidende Rolle spielen. ${ }^{35}$

Mehrere Schritte in diese Richtung wurden bereits getan. So vertrat der Kunsthistoriker Pierre Francastel (1900-1970) eine Soziologie der Kunst, die der technischen Entwicklung einen entscheidenden Platz zuwies. ${ }^{36}$ Die französische technologie culturelle inspiriert auch historische Arbeiten, die nicht zuletzt kunsthistorische Objekte betreffen, so etwa zur Baugeschichte. ${ }^{37}$ Ferner hat der Anthropologe Alfred Gell in seinem Buch Art and Agency eine Theorie vorgelegt, die als Analyse sozialer Techniken aufgefasst werden kann und zugleich ein breites Spektrum an Objekten einschließt. ${ }^{38}$ Hans Belting hat seinerseits für eine Anthropologie plädiert, welche die Bilder von ihrem physischen Verhältnis zum Mensch her denkt ${ }^{39}$. Nicht zuletzt wird unter dem philosophischen Begriff der Kulturtechnik betont, dass Bild, Schrift und Zahl, die Kultur (im intellektuellen Sinne von Kant) ausmachen, auch als Techniken des Symbolischen zu betrachten sind. ${ }^{40}$ Dies lässt leider die umgekehrte Evidenz außer Acht, dass jede Technik ein symbolisch und eben kulturell durchdrungenes, Kultur (im sozialanthropologischen Sinne) prägendes Phänomen ist. Darüber hinaus bezeichnete das lateinische cultura nicht nur Geistestechniken, sondern auch die Technik der Agrikultur. So riskiert der hybride Begriff der Kulturtechnik die Unterscheidung von Kultur und Technik weiterzutradieren, die er doch eigentlich überwinden möchte. Geschickter wäre wohl zu erkennen, dass Technik und Kultur miteinander verwobene Begriffe sind, die beide auf die operativen und kognitiven Erfahrungen des Menschen mit seiner Umwelt verweisen, und im strengen Sinne synonym sind - dafür erscheint der Ausdruck technologie culturelle bzw. kulturelle Technikwissenschaft nach wie vor passend. So verstanden bietet die Technik eine Perspektive für eine Anthropologie, die ihre Untersuchungsfelder nicht mehr zwischen dem naturwissenschaftlich angehauchten >Technischem Symbole fokussierten `Kulturellem ‘ wählen will, sondern sich zum Anspruch macht, die variierenden Formen des menschlichen Gesellschaftslebens eben jenseits der modernen Unterscheidung zwischen Natur und Kultur zu verstehen. ${ }^{41}$ Es ist in diesem Zusammenhang ein entscheidendes Ergebnis, dass in jüngeren Arbeiten die Diskrepanz zwischen Anthropologie der Technik und Anthropologie der Kunst insofern überwunden wurde, als die Dinge nicht mehr theoretisch zwischen einem konstituierenden Technischen und einem kulturbedingten Ästhetischen zerlegt, sondern ganzheitlich im Bezug auf symbolische Akte erfasst werden. ${ }^{42}$ 


\section{Repräsentationen}

Soll damit die Kunstgeschichte zu einer historischen (Sozial-)Anthropologie der Technik werden ${ }^{43}$ ? Nein, denn Einiges in diesem Forschungsfeld kann sie am besten. Im Spiel ist die Geschichte und die soziale Funktion des abendländischen, modernen Kunst-Begriffs und darüber hinaus die in den Sozialwissenschaften oft debattierte Frage nach dem Verhältnis von Technik und Gesellschaft: Sind Techniken nur Ausdruck der jeweiligen Gesellschaftsformen, oder gestalten sie vielmehr die sozialen Verhältnisse ${ }^{24}$ Nun werden technische Akte selbst jenseits ihrer physischen Ausführung durch Wörter, Mythen, ${ }^{45}$ mentale oder materielle Bilder symbolisch ergriffen, konzeptuell begriffen und kulturell wirksam. Techne in der Antike - wie ars, ingenium oder industria im Mittelalter - bedeutet auch List. Sie impliziert die Verfügung über eine ambivalente Macht, deren Faszination im Prozess der Repräsentation eingefangen werden kann, ohne aufgelöst oder überwunden zu werden. ${ }^{46}$ Techniken rufen also Repräsentationen hervor und werden durch sie sozialisiert: Technikrepräsentationen fügen die Techniken in der Gesellschaft ein und prägen die sozialen Verhältnisse, sie vermitteln zwischen Techniken und Gesellschaften. Das Imaginäre der heutigen Müllentsorgungstechnik besteht zum Beispiel, wie eine ethnographische Studie zeigt, aus Titanenkriegen, Höllenfeuer und Alchimistenschmieden, ${ }^{47}$ wobei sich zeigt, wie geerbte Vorstellungen älterer Mythen, Rituale und Techniken aktualisiert werden. `Leitbilder, wie etwa Wachstum oder Nachhaltigkeit, spielen eine entscheidende Rolle bei technischen Innovationen. ${ }^{48}$ Diese wiederum werden kulturell verarbeitet, unter anderem literarisch: So wird die science-fiction von lebenden Maschinen und genetisch manipulierten Geschöpfen bevölkert. ${ }^{49}$ In diesem Zusammenhang kann auch die Kunstgeschichte einen Beitrag leisten, indem sie ihre empirische Expertise dafür einsetzt, die Materialisierungen von Repräsentationen technischer Objekte und Prozesse zu beschreiben und in ihrer sozialen Relevanz zu verstehen. Drei Beispiele von Werkzeug- bzw. Instrumentbildern, die technische Akte evozieren, um auf soziale Zusammenhänge zu wirken, mögen dies im Folgenden verdeutlichen.

Im südlichen Querschiff der Abteikirche San Miniato al Monte in Florenz ist ein Wandbild vom Beginn des 15. Jahrhunderts zu sehen (Abb. 6), das den auferstandenen Christus zeigt, der mit seiner rechten Hand das neben ihm stehende Holzkreuz umfängt, während er mit der Linken auf seine Seitenwunde deutet. Etwa fünfundzwanzig Werkzeuge sind ringsum angeordnet, wobei deren scharfen oder spitzen Enden allesamt auf seinen nackten Körper gerichtet sind. Dieser und das Kreuz wurden a fresco gemalt, die Werkzeuge aber wurden mittels anderer Techniken, nämlich a secco und durch Metallblatt-Applikationen, hinzugefügt. So wirken die Werkzeuge sowohl durch den Herstellungsprozess als auch innerhalb der Bildlogik als Fremdkörper; Obwohl heute meist nur noch die Umrisse ihrer metallenen Teile zu erkennen sind, ist ihre bedrohliche Präsenz auf dem einst ganz blauen Hintergrund 


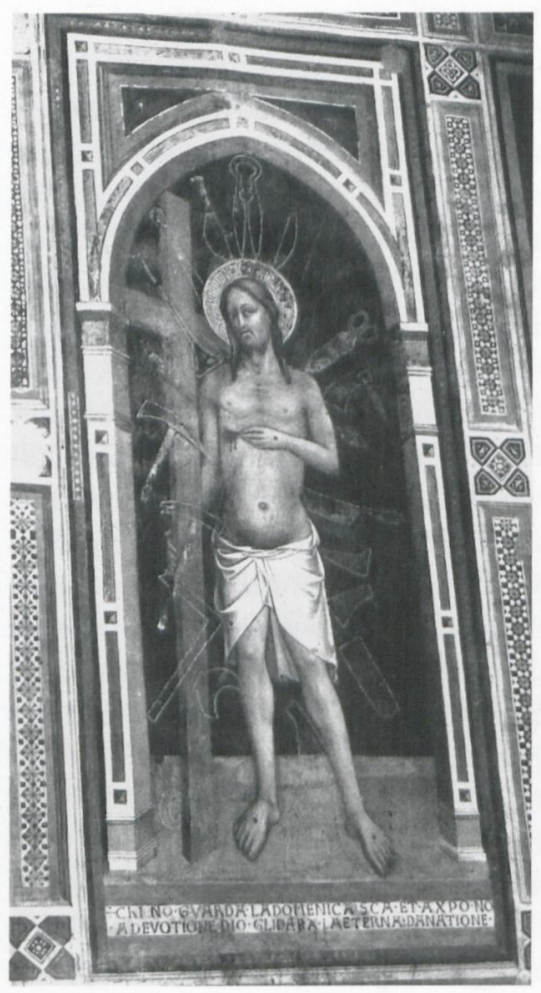

6. Feiertagschristus, Fresko, Florenz, San Miniato al Monte, Mariotto di Cristofano, Frühes 15. Jahrhundert

leicht vorstellbar. Eine gut lesbare und leicht memorierbare Inschrift warnt den Betrachter in der Volkssprache: chi no guarda la domenica sca et a xpo no a devotione dio gli dara la eterna danatione - "wer den heiligen Sonntag nicht respektiert und Christus nicht ehrt, den wird Gott für ewig verdammen«. Jeder wird gemahnt, seine eigenen Werkzeuge am Sonntag ruhen zu lassen, andernfalls würde man symbolisch das Leiden des Erlösers fortführen und dafür bestraft werden - denn jedes tätige Werkzeug würde direkt auf den Leib Christi einwirken und diesen abermals martern, wie im verwandten Bildtypus der Arma Christi. Die Gemeinschaft wird hier sowohl über die Verwendung diverser Werkzeuge definiert, die als Repräsentanten verschiedener Berufe wie Schneider, Metzger, Zimmermann usw. fungieren, als auch über das Aussetzen der Tätigkeiten, um sich in der Kirche für den Gottesdienst und die Eucharistie zu versammeln. Das Bild wurde auf der einzigen Wand der Kirche gemalt, von welcher der Blick durch das Hauptportal der Kirche, wenn es geöffnet ist, bis zur sich damals im Bau befindlichen Domkuppel schweifen kann. Dies ist wohl kein Zufall, denn dort wurde eben auch an Feiertagen gearbeitet, beispielsweise um die Mauern feucht zu halten, wie Archiveinträge aus den Sommern 1427 und 1428 bezeugen. ${ }^{50}$ Auf diesen Regelbruch inmitten der Stadt wird man in San Miniato mit diesem Bild reagiert haben, dessen 

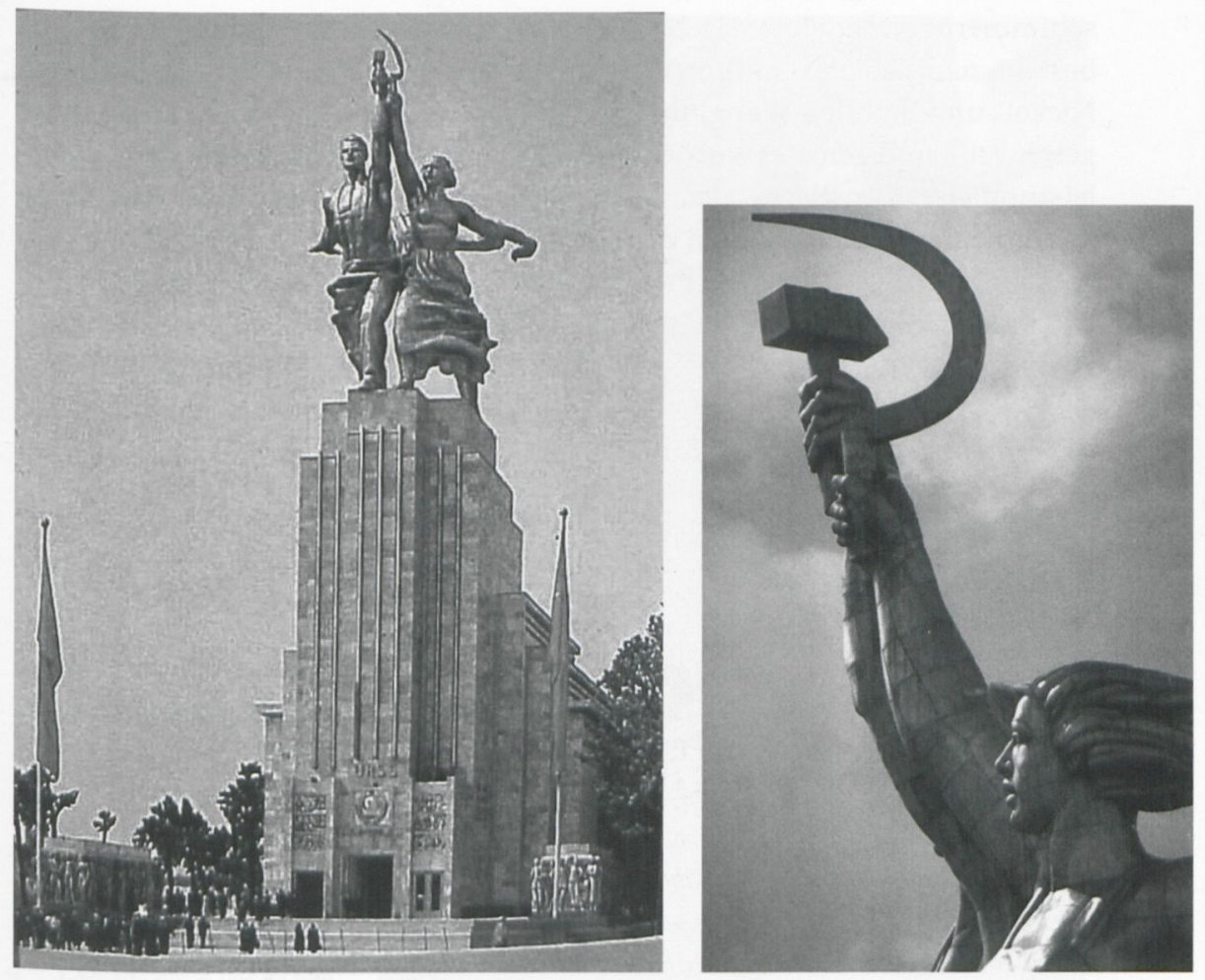

7. Sowjetischer Pavillon aufder Pariser Weltausstellung von 1937

8. Vera Mukhina, Arbeiter und Kolchosbäuerin, 1937, Detail

Ikonographie sonst vor allem in ländlichen Pfarreien der Alpen begegnet und dessen Inschrift unter allen bekannten Bildern des Feiertagschristus die Warnung am strengsten formuliert. ${ }^{51}$ Die große Schere über dem Kopf Christi sollte ebenfalls auf den konkreten Anlass bezogen sein, als Verweis auf die Wollweberzunft, die für den Dombau von der Florentiner Kommune beauftragt und damit in letzter Instanz verantwortlich war.

Eine weitere Verbildlichung des Zusammenhanges von Technik und Gesellschaft stellen die Figuren eines Arbeiters und einer Kolchosbäuerin dar, welche Vera Mukhina für den sowjetischen Pavillon auf der Pariser Weltausstellung bzw. Exposition Internationale des Arts et Techniques dans la Vie Moderne von 1937 entwarf (Abb. 7-8). ${ }^{52} \mathrm{Ihr}$ Bedeutungsgehalt wird bereits durch die Größenverhältnisse herausgestellt: Die Turmfassade vor der sich lang nach hinten streckenden Ausstellungshalle ist fünfunddreißig Meter hoch, die Doppelskulptur darüber zwanzig. Das Ganze kulminiert in den Werkzeugen, welche der Arbeiter und die Kolchosbäuerin triumphierend emporhalten: Hammer und Sichel, die handwerklichen Embleme der Sowjetunion, deren heraldische Silhouette sich gegen den Himmel abzeichnet, und die in ihrer 
schimmernden Stahloberfläche geradezu transzendiert wirken. Die Gruppe besteht nämlich aus millimeterdünn getriebenen Platten einer rostfreien Nickel- und Stahllegierung, die, wie in der Aerotechnik üblich, aneinander geschweißt und genietet wurden. Sie beweist sich damit als kollektive Arbeitsleistung der Künstlerin, des Architekten und der Schwerindustrie: Diese monumentalen Bilder sollten durch ihr Material und ihren Herstellungsprozess die Verschränkung von Produktionsbedingungen und Gesellschaftsmodell veranschaulichen. Dabei wurde mit den Werkzeugen auf Objekte rekurriert, die gerade infolge der hier gefeierten Mechanisierung ihre Funktion eingebüßt hatten, und eine Form gewählt, die, indem sie die Siegesallegorien der griechischen Antike aufruft, die kanonisierte Kunsttradition aktualisiert. So wurde ein sozialutopisches, skunstindustrielles` Bild des Handwerks geschaffen, das die Folgen seiner Verdrängung zugunsten der industriellen Produktion einerseits, der künstlerischen Tätigkeit andererseits zu überwinden behauptete.

Ein smaterialikonographischer ${ }^{53}$ bzw. stechnikikonologischer ${ }^{3}$ Zugang eignet sich also besonders für die Analyse von Technikrepräsentationen. Darüber hinaus legen sie der Kunstgeschichte nahe, nach dem historisch variierenden Verhältnis von Technik und Kunst zu fragen, sowie nach dem Status der Kunstgeschichtschreibung als einer zwar spezifischen, aber nichtsdestoweniger prägenden Meistererzählung der abendländischen Technikgeschichte. ${ }^{54}$ Beide Fragen verweisen in die italienische Renaissance`, als Künstlerbiographien entstanden, die bis heute grundlegend für die kunstwissenschaftliche Forschung blieben, und Kunsttheorien entwickelt wurden, die innerhalb der künstlerischen Arbeit das Technisch-Materielle herabsetzten. So inaugurierte Federico Zuccari 1578 eine repräsentative Fassade für sein neu gebautes Atelier in Florenz (Abb. 9), die drei Reliefs aufweist: Dort sind diverse Werkzeuge und Instrumente so dargestellt, als seien sie im Stein gefangen. Wären sie ganz freigelegt, würden sie, gleichsam durch die Ateliermauer herausgeschleudert, auf die Passanten herunter stürzen. Diese Anspielung auf die non finito-Ästhetik Michelangelos (1475-1564) diente wohl als Gegenbild zu dem überlegenen disegno, kann man doch davon ausgehen, dass der große, leicht zum Betrachter geneigte, heute leere Steinrahmen im Obergeschoss für eine Allegorie des Zeichnens vorgesehen war. ${ }^{55}$ So wurde die Zeichenkunst als eine über jeder anderen Technik stehenden »Metatechnik « ${ }^{56}$ definiert und zelebriert, die weniger auf routinierter Praxis beruht, als darauf, virtuos und unmittelbar mentale Bilder zu vermitteln. Der Kontrast zwischen den rohen und glatten Steinquadern im unteren Bereich und den Backsteinen im oberen Teil verweist dabei sowohl auf die römische Palastarchitektur der Zeit als auch auf die Beherrschung von natura durch ars. Diese Atelierfassade, ostentativ von dem Wohnhaus getrennt, das einst dem berühmten Florentiner Künstler Andrea del Sarto (1486-1530) gehört hatte, war die erste ihrer Art. Ihr intellektueller Anspruch führte die Überlegungen Giorgio Vasaris (15111574) fort, des Autors der Vite de' più eccellenti pittori, scultori e architettori, ${ }^{57}$ der 
9. Fassade des Ateliers von Federico Zuccari, Florenz, 1575-1579

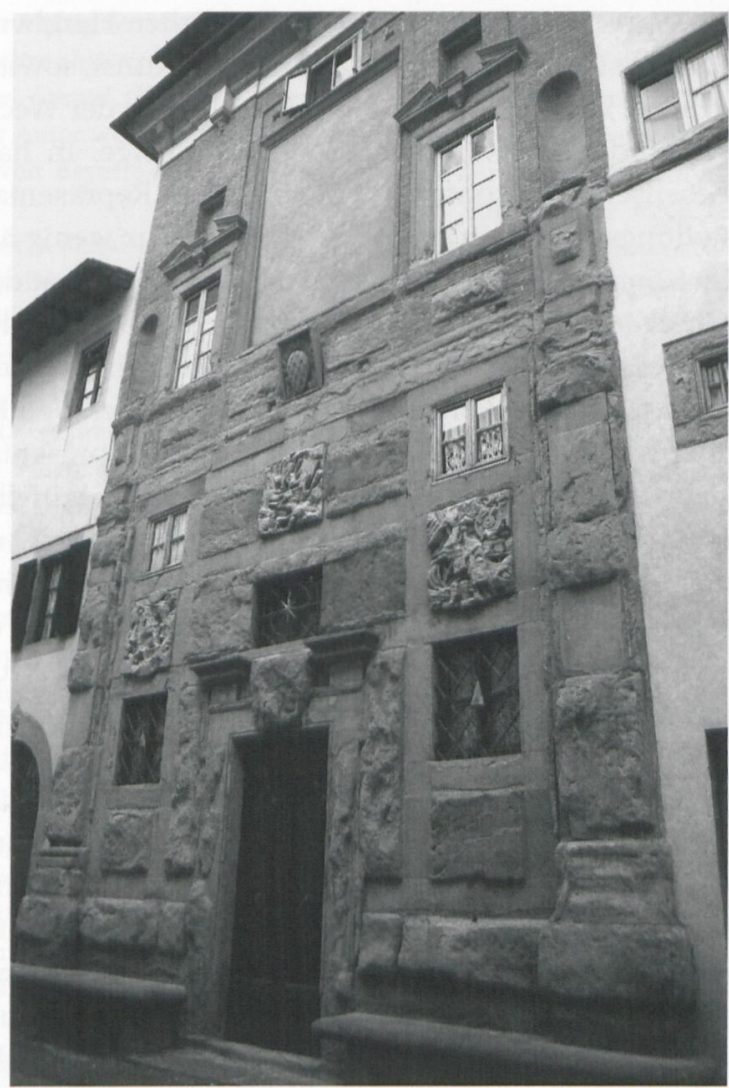

seine eigenen Häuser in Arezzo und Florenz ebenfalls mit kunsttheoretischen Bilder freskiert hatte und dessen Vorhaben der Innenausmalung der Florentiner Domkuppel Zuccari übernommen hatte. Die Werkzeugereliefs hingegen scheinen den Metopen im Innenhof eines unerhört spektakulären Wohnhauses entlehnt zu sein, das der Bildhauer Leone Leoni wenige Jahre zuvor in Mailand hatte bauen lassen. ${ }^{58}$ So öffentlich die Fassade war, betraf jedoch ein solcher Diskurs vor allem das Künstlersubjekt selbst und einen begrenzten Kreis gebildeter Kenner, Auftraggeber und Kollegen, wie sie sich etwa in der 1563 gegründeten Accademia del Disegno zusammenfanden. Darin drückt sich die soziale Implikation der Unterscheidung von »Technik« und »Kunst« aus: Von den »Handwerkern« explizit getrennt, bildeten die »Künstler« nur eine kleinere Gruppe innerhalb der Gesellschaft. Sie waren entsprechend flexibler und innovativer, wie es in Florenz bereits im 15. Jh. besonders deutlich wurde. ${ }^{59}$

Die Zurschaustellung von künstlerischen Werken involvierte zwar nach wie vor die breite Gesellschaft, nun aber viel mehr durch die verschiedenen Techniken der Aneignung als durch eine direkte Beteiligung am Herstel- 
lungsprozess, wie es noch bei alltäglichen Handwerkserzeugnissen der Fall war. Die spezialisierte Produktion von Kunst, sowie und vor allem von industrieller Ware, haben eine Verschiebung in der Wechselwirkung von Techniken und ihrer Repräsentationen zur Folge. In handwerklich dominierten Gesellschaften können nämlich soziale Repräsentationen anhand von Herstellungstechniken entstehen, die sich nur wenig ändern und bekannt sind. Im Kontext von Kunst und Industrie dagegen hat die Produktion von Gesellschaft weniger direkt mit komplexer gewordenen Herstellungstechniken zu tun, als mit ständig erneuerten Objekten, welche die Mehrheit nur noch bewundert und konsumiert. So sind aufwendige Kunstprodukte, Apparate oder technische Geräte zugleich soziale Repräsentationen, welche in ihrem Gebrauch die Herstellung sozialer Formen unmittelbar bedingen. ${ }^{60}$ Techniken und ihre Repräsentationen determinieren einander und konstituieren zusammen die Gesellschaften: In diesem Zusammenhang liegt es nahe, dass die semantische Unterscheidung zwischen Werkzeug und Instrument, die zwischen Handwerk und Kunst oder Wissenschaft trennen will, sich gerade parallel zur fortschreitenden Industrialisierung des Abendlandes radikalisierte, in der das Maschinenwerkzeug triumphierte und die Gültigkeit herkömmlicher technischer Repräsentationen relativierte. Die technische Entwicklung hatte zu einer Neuordnung in den Repräsentationen menschlicher Tätigkeiten und Dinge geführt - eine Situation, die sich bis heute nur verschärft und verkompliziert hat. Damit steckt schließlich in dem Begriffspaar, das diesem Band seinen Titel gibt, eine Aufforderung an die Kunstgeschichte, sich mit Techniken und ihren Repräsentationen auseinanderzusetzen. Dabei geht es nicht bloß um die kritische Analyse des Einsatzes von handwerklichen, mechanischen, chemischen Techniken oder noch von Elektro- und Biotechniken in der Produktion von Kunst, sondern, über den Weg einer historischen Verortung des Kunstbegriffes, vor allem um die Teilhabe der Kunstgeschichte am holistischen Unternehmen der Sozialwissenschaften.

* Ich bedanke mich für ihre sorgfältige Lektüre bei Gianenrico Bernasconi, Matthias Krüger, Katja Müller-Helle, Susanne Pollack und Julia Saviello.

${ }^{1}$ Siehe Jacob Grimm u. Wilhelm Grimm: Deutsches Wörterbuch, 32 Bde., Leipzig 1854-1960, s.v. Instrument, Bd. 10, Sp. 2146-2152, Werkzeug, Bd. 29, Sp. 419-436. Im Französischen etwa leitet sich outil aus utensilia und verweist so ebenfalls auf Nützlichkeit (utilitas): Alain Rey (Hg.): Dictionnaire historique de la langue française [Paris 1992], 3 Bde., Paris 2007, s.v. outil, Bd. 2, S. 2508.

${ }^{2}$ Zur Artikulation beider Begriffe: Matthias Krüger: Das Relief der Farbe. Pastose Malerei in der französischen Kunstkritik. 1850-1890, München u. Berlin 2007, Kap. „Das Handwerk der Malerei«, S. 197-227, bes. S. 206-208.

${ }^{3}$ Zur Geschichte der Bilder als Technik des Wissens: Horst Bredekamp, Matthias Bruhn u. Gabriele Werner (Hg.): Bildwelten des Wissens. Kunsthistorisches Jahrbuch für Bildkritik, seit 2003; Horst Bredekamp, Birgit Schneider u. Vera Dünkel (Hg.): Das Technische Bild. Kompendium zu einer Stilgeschichte wissenschaftlicher Bilder, Berlin 2008. Ferner: Martin Schulz u. Beat Wyss (Hg.): Techniken des Bildes, München 2010.

${ }^{4}$ Samuel von Quiccheberg: Inscriptiones vel tituli theatri amplissimi [München 1565], hg., übers. 
u. komm. von Harriet Roth: Der Anfang der Museumslehre in Deutschland. Das Traktat "Inscriptiones vel tituli theatri amplissimi« von Samuel Quiccheberg, Berlin 2000, S. 60-69; der Autor wollte mit seinem Traktat dem Herzog von Bayern seine Dienste anbieten.

${ }^{5}$ Heute im Musée national de la Renaissance, Écouen. Siehe Manfred Welker: Die Reichsstadt Nürnberg, ein Zentrum des Schmiedeeisen verarbeitenden Handwerks, in: Hermann Maué u. a. (Hg.): Quasi centrum Europae. Europa kauft in Nürnberg. 1400-1800, Ausst.-Kat. (Nürnberg, Germanisches Nationalmuseum), Nürnberg 2002, S. 117-137, hier 119-125 zu Leonhard Danner, seiner Drahtziehbank und seiner Erfindung der "Brechschraube " um 1550, die ebenfalls Eingang in der Dresdner Kunstkammer fand und auch von Quiccheberg mit der Kategorie instrumenta violenta erwähnt wird. Zur Adelung des Handwerks ab dem 16. Jahrhundert: Klaus Maurice: Der drechselnde Souverän. Materialien zu einer fürstlichen Maschinenkunst, Zürich 1985. Zur Dresdner Kunstkammer, die vor allem aus Wissenschaftsinstrumenten und Werkzeugen bestand, Michael Korey: Die Dresdner Kunstkammer. Instrumente des Wissens und des Könnens, in: Jahrbuch der Staatlichen Kunstsammlungen Dresden, 30, 2002/ 03 (2006), S. 47-60.

${ }^{6}$ Michael Praetorius: Syntagma Musicum. De Organographia, Wolfenbüttel 1619-1620; siehe Conny Restle: Organologie. Die Kunde von den Musikinstrumenten im 17. Jahrhundert, in: Helmar Schramm, Ludger Schwarte u. Jan Lazardzig (Hg.): Instrumente in Kunst und Wissenschaft. Zur Architektonik kultureller Grenzen im 17. Jahrhundert, Berlin 2006, S. 277-289; Florence Gétreau (Hg.), Les collections d'instruments de musique = Musique. Images. Instruments. Revue française d'organologie et d'iconographie musicale, 8, 2006 u. 9, 2007.

7 Siehe Michael Heidelberger: Experiment und Instrument, in: Schramm/Schwarte/Lazardzig 2006 (wie Anm. 6), S. 378-397. Ferner: Bart Grob u. Hans Hooijmaijers (Hg.): Who Needs Scientific Instruments? Conference on Scientific Instruments and their Users, Leiden 2006; Giorgio Strano u. a. (Hg.): European Collections of Scientific Instruments, 1550-1750, Leiden u. a. 2009.

${ }^{8}$ Siehe auch zur operativen Virtuosität Pamela H. Smith: The Body of the Artisan. Art and Experience in the Scientific Revolution, Chicago 2004.
${ }^{9}$ Das Objekt wurde im Katalog der Sammlung veröffentlicht (Willum Worm (Hg.): Museum Wormianum, Leiden 1655) und mit ihr nach Worms Tod dem dänischen König übergeben. Es befindet sich heute in der königlichen Kunstkammer in Kopenhagen. Ich bedanke mich bei Lisbet Tarp für ihren freundlichen Hinweise.

${ }^{10}$ Heute mit anderen Musikinstrumenten aus Marmor in der Galleria Estense in Modena. Siehe Maria Grazia Bernardini (Hg.): Un cembalo in marmo per Francesco II d'Este, Modena 2005.

${ }^{11}$ Denis Diderot $\mathrm{u}$. Jean le Rond D'Alembert (Hg.): Encyclopédie ou Dictionnaire raisonné des sciences, des arts et des métiers, 28 Bde., Paris 17511772. Siehe Barbara Holländer: Technik und Arbeit in den Tafelbänder der Encyclopédie, in: Hans Holländer (Hg.): Erkenntnis, Erfindung, Konstruktion. Studien zur Bildgeschichte von Naturwissenschaften und Technik vom 16. bis zum 19. Jahrhundert, Berlin 2000, S. 789-806.

${ }^{12}$ Siehe über Technik- und Wissenschaftsmuseen Helmut Trischler: Das Technikmuseum im langen 19. Jahrhundert: Genese, Sammlungskultur und Problemlagen der Wissenskommunikation, in: Bernhard Graf u. Hanno Möbius (Hg.): Zur Geschichte der Museen im 19. Jahrhundert 17891918, Berlin 2006, S. 81-92.

${ }^{13}$ Dazu Francesca Lederlin: L'apparition du terme objet d'art: quelques hypothèses socio-culturelles, in: French studies in Southern Africa, 26, 1997, S. 33-41.

${ }^{14}$ Beide Begriffe parallel benutzt z. B. in C. Becker u. Jakob von Hefner: Kunstwerke und Geräthschaften des Mittelalters und der Renaissance, 3 Bde., Frankfurt am Main 1852-1863.

${ }^{15}$ Gottfried Semper: Der Stil in den technischen und tektonischen Künsten oder Praktische Ästhetik. Ein Handbuch für Techniker, Künstler und Kunstfreunde, 2 Bde. [Frankfurt am Main u. Zürich 18601863], Hildesheim 2008. Vgl. auch ders., The Ideal Museum. Practical Art in Metals and Hard Materials [Manuskript 1852], hg. von Peter Noever, Wien 2007. Für eine historiographische Übersicht an der Schwelle von Kunstgewerbemuseum und Kunstwissenschaft siehe Michael Conforti: Les musées des arts appliqués et l'histoire de l'art, in: Edouard Pommier (Hg.): Histoire de l'histoire de l'art, Paris 1997, S. 327-347.

${ }^{16}$ Alois Riegl: Volkskunst, Hausfleiss und Hausindustrie [Berlin 1894], Mittenwald 1978; dazu Stefan Muthesius: Alois Riegl: Volkskunst, Haus- 
fleiss und Hausindustrie, in Richard Woodfield (Hg.): Framing Formalism. Riegls' Work, Amsterdam 2001, S. 135-150; Georg Vasold: Alois Riegl und die Kunstgeschichte als Kulturgeschichte. Überlegungen zum Frühwerk des Wiener Gelehrten, Freiburg im Breisgau 2004. Siehe auch Katalin Sinkó: Die Entstehung des Begriffs der Volkskunst in den Kunstgewerbemuseen des Zeitalters des Positivismus: Ornament als Nationalsprache, in: Acta historiae artium, 46, 2005, S. 205-259. Zum »Kunstwollen«: Alois Riegl: Spätrömische Kunstindustrie [Wien 1901], Berlin 2000.

${ }^{17}$ Siehe der Beitrag von Gottfried Korff in diesem Band; allgemeiner ders.: Kulturforschung im Souterrain. Aby Warburg und die Volkskunde, in: Kaspar Maase u. Bernd-Jürgen Warneken (Hg.): Unterwelten der Kultur. Themen und Theorien der volkskundlichen Kulturwissenschaft, Köln, Weimar u. Wien 2003, S. 143-178.

${ }^{18}$ Siehe Henri Focillon: Das Leben der Formen [La vie des formes, Paris 1934], Bern 1954; Annamaria Ducci: Henri Focillon, l'arte popolare e le scienze sociali, in: Annali di critica d'arte, 2, 2006, S. 341-389.

${ }^{19}$ Siehe George Kubler: Die Form der Zeit. Anmerkungen zur Geschichte der Dinge [The Shape of Time. Remarks on the History of Things, New Haven (Conn.) 1962], Frankfurt am Main 1982. Eine umfassende, semiotische Erfassung der zeitgenössischen Sachkultur in der Konsumgesellschaft bietet wenige Jahre später Jean Baudrillard: Le système des objets [Paris 1968], Das System der Dinge. Über unser Verhältnis zu den alltäglichen Gegenständen, Frankfurt am Main 2007.

${ }^{20}$ Jacques Boucher de Perthes: Antiquités celtiques et antédiluviennes. Mémoire sur l'industrie primitive et les arts à leur origine, Paris 1847-1864. $\mathrm{Zu}$ den Konsequenzen prähistorischer Entdeckungen für die Kunstgeschichte: Ulrich Pfisterer: Altamira - oder Die Anfänge von Kunst und Kunstwissenschaft, in: Vorträge aus dem WarburgHaus, 10, 2007, S. 13-80.

${ }^{21}$ Siehe François Sigaut: De la technologie à l'évolutionnisme, l'œuvre de Pitt-Rivers, in: Gradhiva, 8, 1990, S. 20-37.

${ }^{22}$ Ernst Kapp: Grundlinien einer Philosophie der Technik. Zur Entstehungsgeschichte der Cultur aus neuen Gesichtspunkten, Braunschweig 1877; siehe Harald Leinenbach: Die Körperlichkeit der Technik. Zur Organprojektionstheorie Ernst Kapps, Essen 1990.
${ }^{23}$ Marcel Mauss: Les techniques du corps, in: Journal de Psychologie, 32/3-4, 1935, S. 271-293, Körpertechniken, in: ders., Soziologie und Anthropologie, 2 Bde., Frankfurt am Main 1981-1989, Bd. 2, S. 199-220. Siehe auch François Sigaut: Les outils et le corps, in: Thierry Pilon u. Georges Vigarello (Hg.): Corps et techniques = Communications, 81, 2007, S. 9-30.

${ }^{24}$ André Leroi-Gourhan: L'Homme et la matière, Paris 1943; ders.: Milieu et techniques, Paris 1945; ders.: Hand und Wort. Die Evolution von Technik, Sprache und Kunst [Le Geste et la Parole, 1. Technique et langage, 2. La mémoire et les rythmes, Paris 1964-1965], Frankfurt am Main 1988. Siehe Françoise Audouze u. Nathan Schlanger (Hg.): Autour de l'homme. Contexte et actualité d'André LeroiGourhan, Antibes 2004. Für eine nuancierte Analyse des Evolutionismus von Leroi-Gourhan und zum Konzept der technischen Evolution, Nathan Schlanger: Piaget et Leroi-Gourhan. Deux conceptions biologiques des connaissances et des techniques, in: Bruno Latour u. Pierre Lemonnier (Hg.): De la préhistoire aux missiles balistiques. L'intelligence sociale des techniques, Paris 1994, S. 165-186; Xavier Guchet: Évolution technique et objectivité technique chez Leroi-Gourhan et Simondon, in: Revue Appareil, 2, 2008, http://revues.mshparisnord.org/appareil/index.php?id= 580 [22.08.10].

${ }^{25}$ Siehe die Zeitschrift Techniques \& culture. Revue semestrielle d'anthropologie, seit 1983; François Sigault: Préface. Georges Haudricourt et la technologie, in: Georges Haudricourt: La Technologie science humaine. Recherches d'histoire et d'ethnologie des techniques, Paris 1987, S. 9-34; Hélène Balfet (Hg.): Observer l'action technique. Des chaînes opératoires pour quoi faire?, Paris 1991; Pierre Lemonnier: Elements for an Anthropology of Technology, Ann Arbor 1992; ders. (Hg.): Technological Choices. Transformation in Material Cultures Since the Neolithic, London 1993; Robert Cresswell: Prométhée ou Pandore? Propos de technologie culturelle, Paris 1996. Mit einen hauptsächlich historischen Zugang: Technology and Culture. The International Quarterly of the Society for the History of Technology, seit 1959.

${ }^{26}$ Siehe für eine Zusammenarbeit zwischen Anthropologen und Soziologen der Technik Latour/Lemonnier 1994 (wie Anm. 24); ebd. Madeleine Akrich: Comment sortir de la dichotomie technique/société. Présentation des diverses so- 
ciologies de la technique, S. 105-131. Siehe auch Werner Rammert u. Cornelius Schubert (Hg.): Technografie. Zur Mikrosoziologie der Technik, Frankfurt a. M. 2006; Werner Rammert: Technik - Handeln - Wissen. Zu einer pragmatistischen Technikund Sozialtheorie, Wiesbaden 2007.

${ }^{27}$ Siehe Jean-Pierre Warnier: Construire la culture matérielle. L'homme qui pensait avec ses doigts, Paris 1999; Marie-Pierre Julien u. Jean-Pierre Warnier (Hg.): Approches de la culture matérielle. Corps à corps avec l'objet, Paris 1999; Marie-Pierre Julien u. Céline Rosselin: La culture matérielle, Paris 2005; dies. (Hg.), Le sujet contre les objets... tout contre. Ethnographies de cultures matérielles, Paris 2009. Siehe auch die Studien zur Technikerfahrung im »Forschungskolleg Kulturwissenschaftliche Technikforschung « am Institut für Volkskunde und Kulturanthropologie der Universität Hamburg (seit 2008).

${ }^{28}$ Siehe Richard Sennet: Handwerk [The Craftsman, Yale 2008], Berlin 2008; Matthew B. Crawford: Ich schraube, also bin ich. Vom Glück, etwas mit den eigenen Händen zu schaffen [Shop Class as Soulcraft. An Inquiry Into the Value of Work, New York 2009], Berlin 2010. Siehe auch zum Köperbewusstsein: Richard Shusterman: Body Consciousness. A Philosophy of Mindfulness and Somaesthetics, New York 2008.

${ }^{29}$ Bruno Latour: Von der Realpolitik zur Dingpolitik oder: Wie man Dinge öffentlich macht, Berlin 2005.

${ }^{30}$ Die Technikphilosophie hat sich vornehmlich den zeitgenössischen Techniken zugewandt: siehe Christoph Hubig, Alois Huning u. Günter Ropohl (Hg.): Nachdenken über Technik. Die Klassiker der Technikphilosophie, Berlin 2000; Bernhard Irrgang: Philosophie der Technik, Bd. 1 Technische Kultur. Instrumentelles Verstehen und technisches Handeln, Bd. 2 Technische Praxis. Gestaltungsperspektiven technischer Entwicklung, Bd. 3 Technischer Fortschritt. Legitimitätsprobleme innovativer Technik, Paderborn u.a. 2001-2002; sowie die Buchreihe Technikphilosophie, hg. von Klaus Kornwachs, bisher 19 Bde., Münster u. London 2000.

${ }^{31}$ Siehe Klaus Kornwachs (Hg.): Technik - System - Verantwortung, Münster u. London 2004, dort bes. ders.: Technik - System - Verantwortung. Eine Einführung, S. 23-43, u. Walther Ch. Zimmerli: Technik und Philosophie - 125 Jahre, und wie weiter?, S. 665-678.
${ }^{32}$ Siehe beispielsweise www.greenpeace.de.

${ }^{33}$ Siehe als Begegnung von Kunstgeschichte und Anthropologie (nicht nur der Kunst) Thierry Dufrêne u. Anne-Christine Taylor (Hg.): Histoire de l'art et anthropologie, 2009, http://actesbranly. revues.org/60 [24/08/10], Texte z.T. gedruckt in ders. (Hg.): Cannibalismes disciplinaires. Quandl'histoire de l'art et l'anthropologie se rencontrent, Paris 2009.

${ }^{34}$ Vgl. etwa im Französischem: Alain Rey (Hg.): Dictionnaire historique de la langue française [Paris 1992], 3 Bde., Paris 2007, s.v. esthétique, Bd. 1, S. 1311-1312, technique, Bd. 3, S. 3772-3773.

${ }^{35}$ Für die Kunstgeschichte siehe bereits die Skizze von Wolfgang Braunfels, zunächst als Vortrag an der Technischen Hochschule Aachen vorgestellt: Die moderne Kunst und der technische Fortschritt, in: Jahrbuch für Aesthetik und allgemeine Kunstwissenschaft, 6, 1961, S. 17-43.

${ }^{36}$ Siehe Pierre Francastel: Art et technique aux XIXe et XXe siècles [Paris 1956], Paris 1998; Zur Soziologie der »figurativen Techniken« seit dem Quatrocento ders.: Études de sociologie de l'art [Études de sociologie de l'art. Création picturale et société, Paris 1970], Paris 1989; dazu Alain Schnapp u. Pierre Lemonnier: André Leroi-Gourhan et Pierre Francastel, in: Dufrêne / Taylor 2009 (wie Anm. 33).

${ }^{37}$ Siehe Jean-Claude Bessac: L'outillage traditionnel du tailleur de pierre de l'Antiquité à nos jours, Paris 1986; David Morel: Archéologie du bâti et anthropologie. L'exemple du technicien dans le Massif central et sur ses marges ( $\mathrm{XI}^{\mathrm{e}}-\mathrm{XIII}{ }^{\mathrm{e}}$ siècles), in: Elisa Brilli, Pierre-Olivier Dittmar u. Blaise Dufal (Hg.): Faire l'anthropologie historique du Moyen Âge $=$ L'Atelier du Centre de recherches historiques. Revue électronique du CRH, 7, 2010, http://acrh.revues.org/index1911.html [22.08.10]. Siehe auch den Bd. 78 der Typologie des sources $d u$ Moyen Age occidental: Johan David: L'outil, Turnhout 1997.

${ }^{38}$ Alfred Gell: Art and Agency. An Anthropological Theory, Oxford 1998. Vgl. Robin Osborne u. Jeremy Tanner (Hg.): Art's Agency and Art History. New Interventions in Art History, Oxford 2007.

${ }^{39}$ Hans Belting: Bild-Anthropologie. Entwürfe für eine Bildwissenschaft, München 2001.

${ }^{40}$ Siehe Sybille Krämer: Technik als Kulturtechnik. Kleines Plädoyer für eine kulturanthropologische Erweiterung des Technikkonzeptes, in: Kornwachs 2004 (wie Anm. 31), S. 157-164; 
dies. u. Horst Bredekamp, Kultur, Technik, Kulturtechnik: Wider die Diskursivierung der Kultur, in: dies. u. ders. (Hg.): Bild - Schrift - Zahl [München 2003], München 2008, S. 11-22; Harun Maye: Was ist eine Kulturtechnik?, in: Zeitschrift für Medien- und Kulturforschung, 1, 2010 (= Kulturtechnik), S. 121-135.

${ }^{41}$ Siehe Philippe Descola: Par delà nature et culture, Paris 2005, der seine Untersuchung zu den Sozialisierungsformen der $>$ Natur $<$ vor allem auf der kognitiven Ebene entwickelt. Siehe jedoch für eine ikonologische Anwendung im Sinne einer $»$ Anthropologie der Figuration«: ders. (Hg.): La Fabrique des images. Visions du monde et formes de la représentation, Ausst.-Kat. (Paris, Musée du Quai Branly), Paris 2010.

${ }^{42}$ Siehe die Forschungsübersicht und den Vorschlag einer "prozessualen Definition des Stils« von Bruno Martinelli: Style, technique et esthétique en anthropologie, in: ders. (Hg.): L'interrogation du style. Anthropologie, technique et esthétique, Aix en Provence 2005, S. 19-48; dort auch zum Zusammenhang von Stil und Werkzeug Salvatore D'Onofrio: Chaque chose a son "galbe«. Style, technique et esthétique à propos d'outils siciliens, S. 87-96. Ein weiterer anthropologischer Forschungsbericht in Schnapp/Lemonnier 2009 (wie Anm. 36), S. 14-24.

${ }^{43}$ Zur »historischen Anthropologie« französischer Art siehe zuletzt Brilli/Dittmar/Dufal 2010 (wie Anm. 37).

${ }^{44} \mathrm{Zu}$ dieser Diskussion siehe z. B. Georges Guille-Escuret (Hg.): Efficacité technique, efficacité sociale $=$ Techniques $\mathcal{E}$ Culture, 40, 2003, darin besonders Robert Cresswell: Geste technique, fait social total. Le technique est-il dans le social ou face à lui?, S. 125-152.

45 Siehe zu semantischen und narrativen Technikdarstellungen Jean-Luc Jamard ( $\mathrm{Hg}$.): $M y$ thes. L'origine des manières de faire $=$ Techniques $\mathcal{E}$ Culture, 43-44, 2004; Salvatore d'Onofrio u. Frédéric Joulian (Hg.): Dire le savoir-faire. Gestes, techniques et objets = Cahiers d'anthropologie sociale, 1 , 2006; und auch der Beitrag von François Poplin in diesem Band.

${ }^{46}$ Siehe zum Begriff der Repräsentation bzw. représentation aus der Sicht des Historikers: JeanClaude Schmitt: Représentations, in: Claudie Duhamel-Amado (Hg.): Georges Duby. L'écriture de l'histoire, Brussel 1996, S. 267-278; für einen anthropologischen und ikonologischen Zugang
Daniel Russo: Anthropologie et Iconologie. Réflexions sur les apports de Jack Goody à l'analyse de la notion de représentation<, in: Bulletin du centre d'études médiévales d'Auxerre, 2008, http:// cem.revues.org/index4242.html [24.08.10] (ausgehend von Jack Goody: Representations and Contradictions. Ambivalence Towards Images, Theatre, Fiction, Relics and Sexuality, London 1997).

${ }^{47} \mathrm{Zu}$ Technik und Kultur des Entsorgens: Sonja Windmüller: Die Kehrseite der Dinge. Müll, Abfall, Wegwerfen als kulturwissenschaftliches Problem, Münster 2004. Ebenso Technik und Repräsentationen verbindend: Christiane Raynaud: $₫ \grave{A}$ la hache! "Histoire et symbolique de la hache dans la France médiévale (XIIIe-XVe siècles), Paris 2002.

${ }^{48}$ Siehe Nicole C. Karafyllis: Nachwachsende Rohstoffe - Technikbewertung zwischen den Leitbildern Wachstum und Nachhaltigkeit, Opladen 2000; dies.: Zum Systemverständnis von Leitbildern in der Technikentwicklung und -gestaltung, in: Kornwachs 2004 (wie Anm. 31), S. 485-498. Vgl. auch Patrice Flichy: L'imaginaire d'Internet, Paris, 2001.

${ }^{49}$ Siehe Monika Margarethe Raml: Der »homo artificialis « als künstlerischer Schöpfer und künstliches Geschöpf. Gentechnologie in Literatur und Leben, Würzburg 2009. Zur kulturellen Einarbeitung der »technischen Objekte«: Gilbert Simondon: $D u$ mode d'existence des objets techniques [Paris 1958], Paris 2001.

${ }^{50}$ Die Kuppel wurde 1418-1436 erbaut; Feiertagsarbeit zu diversen Zwecken ist zwischen 1420 und 1426 in dreizehn Einträgen belegt: vgl. Margaret Haines: The Years of the Cupola: Digital archive of the sources of the Opera di Santa Maria del Fiore, 1417-1436, Firenze 2002, http://duomo. mpiwg-berlin.mpg.de [09.07.10], s.v. festivas, festivis, festivo, und auch Wolfgang Braunfels: Drei Bemerkungen zur Geschichte und Konstruktion der Florentiner Domkuppel, in: Mitteilungen des Kunsthistorischen Institutes in Florenz, 11/4, 1965, S. 203-226, hier 218: Der Autor von Mittelalterliche Stadtbaukunst in der Toskana [Berlin 1953], Berlin 1979, kennt aus dem italienischen Quattrocento keine einzige weitere Lizenz zur Sonntagsarbeit. Ich danke Alexander Markschies für diese Referenzen. Die Datierung des Bildes durch seine Funktion verstärkt seine stilistiche Einordnung als ein Frühwerk von Mariotto di Cristofano (1393-1457): Siehe Angelo Tartuferi: Le opere d'arte e la decorazione pittorica della chiesa, in: 
Francesco Gurrieri, Luciano Berti u. Claudio Leonardi (Hg.): La Basilica di San Miniato al Monte a Firenze, Firenze 1988, S. 183-214, hier 208.

${ }^{51}$ Siehe Dominique Rigaux: Le Christ du dimanche. Histoire d'une image médiévale, Paris 2005, S. 99,154 u. 302-303 zu diesem Bild (mit Fehldeutungen der Maltechnik und des Konservierungszustandes).

${ }^{52}$ Das Werk ist heute in Moskau ausgestellt. Ich danke Monika Wagner und einer Gruppe Hamburger Studenten für gemeinsame Überlegungen im Rahmen eines Seminars. Siehe OttoKarl Werckmeister: Vera Muchina, Arbeiter und Kolchosbäuerin, in: Kunsthistorische Arbeitsblätter, 2002/2, S. 41-50; Bettina Jungen: Kunstpolitik versus Kunst. Leben und Werk der Bildhauerin Vera Muchina (1889-1953), Bielefeld 2005.

${ }^{53}$ Siehe zur Materialsemantik in der Kunst Thomas Raff: Die Sprache der Materialien. Anleitung zu einer Ikonologie der Werkstoffe, München 1994; für die moderne und zeitgenössische Kunst Monika Wagner: Das Material der Kunst. Eine andere Geschichte der Moderne, München 2001; dies., Dietmar Rübel u. Sebastian Hackenschmidt (Hg.): Lexikon des künstlerischen Materials. Werkstoffe der modernen Kunst von Abfall bis Zinn [München 2002], München 2010.

${ }^{54}$ Siehe für eine Pionierarbeit Ernst Kris u. Otto Kurz: Die Legende vom Künstler. Ein historischer Versuch, Wien 1934. Allgemeiner über technische Narrationen im Zusammenhang mit der Geschichte der Technik im Abendland, jedoch ohne Einbeziehung der Kunstgeschichtsschreibung: François Sigaut: Les techniques dans la pensée narrative, in: Jamard 2004 (wie Anm. 45), S. 191-214.

${ }_{55}$ Die stark verwitterten Reliefs wurden 2002-2003 rekonstruiert und sind nicht ganz als historische Zeugnisse zu betrachten. Ob das ausgehend von anderen Werken Zuccaris supponierte, gemalte Bild jemals realisiert wurde, ist ungewiss. Siehe Cristina Acidini: Un episodio fiorentino: le formelle dello Studio Zuccari nella facciata-manifesto, in: dies. u. Elena Capretti (Hg.): Innocente e calunniato. Federico Zuccari (1539/
40-1609) e le vendette d'artista, Firenze 2009, S. 164171.

${ }^{56}$ Siehe Robert Williams: Art, Theory, and Culture in Sixteenth-Century Italy. From Techne to Metatechne, Cambridge 1997, S. 135-150 zur Theorie Zuccaris.

${ }^{57}$ Siehe ebd., S. 29-72; Giorgio Vasari: Lebensbeschreibungen der berühmtesten Maler, Bildhauer und Architekten [Vite de' più eccellenti pittori, scultori e architettori 2., revidierte Ausgabe Firenze 1568], hg. von Alessandro Nova u.a. Berlin 2004.

${ }^{58}$ Siehe Hans-Peter Schwarz: Das Künstlerhaus. Anmerkungen zur Sozialgeschichte des Genies, Braunschweig 1990, S. 177-182, 194-200; Hartmut Olbrich: Die Casa Zuccari in Florenz: Genese und Erscheinung eines Künstlerhauses der Renaissance, Bamberg 1999, S. 135-138. Zum Topos des Künstlerateliers: Michael Cole u. Mary Pardo (Hg.): Inventions of the Studio. Renaissance to Romanticism, Chapell Hill u. London 2005; Michael Diers u. Monika Wagner (Hg.): Topos Atelier. Werkstatt und Wissensform, Berlin 2010 (Hamburger Forschungen zur Kunstgeschichte, 7).

${ }^{59}$ Siehe etwa Édouard Pommier: Comment l'art devient l'Art dans l'Italie de la Renaissance, Paris 2007. Über solche Situationen beschleunigter Interaktionen zwischen Sozialem und Technischem siehe Robert Cresswell: La nature cyclique des relations entre le technique et le social. Approche technologique de la chaîne opératoire, in: Latour/Lemonnier 1994 (wie Anm. 24), S. 275288.

${ }^{60}$ Vgl. die Beobachtung, dass die Bezüge zwischen Techniken und Gesellschaften in traditionellen Kontexten metaphorischer und in industriellen Kontexten unmittelbarer zu sein scheinen, wobei der Anthropomorphismus, zuerst projiziert oder figuriert, nun win die Mechanismen selber« eindringen würde: Bruno Latour u. Pierre Lemonnier: Introduction. Genèse sociale des techniques, genèse technique des humains, in: Latour/Lemonnier 1994 (wie Anm. 24), S. 9-24, hier 18. 